\title{
Masculinidad-feminidad y salud mental
}

\author{
Rolando Díaz Loving, Sofía Rivera Aragón \& \\ Pedro Wolfgang Velasco Matus \\ Universidad Nacional Autónoma de México
}

Recibido: 30 de mayo del 2012 / Aprobado: 18 de junio del 2012

La masculinidad se reviste de una visión que destaca la acción, la fortaleza, la soberanía, la independencia, el egoismo y la imposición (instrumentalidad y agencia), en tanto la feminidad recoge el lado asociado a las emociones, el cuidado, la ternura, la pasividad y la sumisión (expresividad, afecto y comunión). Se aplicaron a una muestra de 100 hombres y 100 mujeres el Inventario de Masculinidad-Feminidad y el Inventario de Salud Mental. Las correlaciones y regresiones realizadas por separado para hombres y mujeres muestran algunas diferencias por sexo pero un acuerdo general en el que la presencia de una instrumentalidad positiva se vincula con el hecho de ser más asertivos y claramente menos narcisistas, menos paranoides, menos fantasiosos, menos obnubilados, menos solitarios y menos apáticos.

género / personalidad / salud mental

\section{Masculinity-femininity and mental health}

Masculinity is presented in a vision that stresses action, strength, independence, egotism, imposition and abusiveness (instrumental and agentic); while femininity picks up on emotions, care, tenderness, passivity and submissiveness (expressive, affectionate and communal). 100 men and 100 women answered the Masculinity and Femininity Inventory and a Mental Health Inventory. Correlations and regression conducted separately for males and females show certain differences by sex. However, in general, positive instrumentality is related to more assertiveness and less narcissism, less paranoia, less loneliness and more energy.

gender / personality / mental health

Correo electrónico: loving@servidor.unam.mx 
Desde el nacimiento, la construcción del género de las personas tiene una relación estrecha con el desarrollo físico, social y emocional (Díaz Loving \& Rocha Sánchez, 2008). Tanto niños como adultos agrupan de manera selectiva la información que es congruente con su identidad sexual. Prácticamente desde la infancia se desarrollan no solo expectativas, sino también preferencias y conductas que responden a los patrones socioculturales normativos al grado de que, según Erickson (1968), los roles de género se convierten en una tarea central del desarrollo de una persona.

Por su parte, los roles de género son una construcción conformada por todas aquellas expectativas y creencias populares sobre las actividades, los rasgos, las características y los atributos que distinguen a los hombres de las mujeres y viceversa, producto de los estándares o estereotipos socialmente establecidos, adaptados y aceptados para cada entidad genérica (Rocha, 2004). A su vez, los rasgos de instrumentalidad y expresividad constituyen dimensiones atributivas de la personalidad que son vistas como aspectos globales del autoconcepto de un hombre y una mujer y que se componen de características normativas para los géneros (Díaz Loving, Rocha \& Rivera, 2004; Spence \& Helmreich, 1978; Spence, 1993; Stoller, 1968).

En un principio, masculinidad y feminidad fueron entendidos como puntos opuestos de un continuo, por lo 
que se presentaban como contrapartes (Medina, 2007). Las diferencias en las atribuciones y roles asignados a cada sexo se extrapolaban a que cada uno pertenezca a mundos diferentes y complementarios, y es a partir de aquí que surgieron los estereotipos de género (Jayme \& Sau, 2004). Estos estereotipos son creencias exageradas asociadas a cada sexo, que indican cómo debe comportarse cada uno. Desde esta perspectiva tradicional y socioculturalmente estructurada, la masculinidad, según el estereotipo dominante, se asocia al logro, la competitividad, la autonomía, el dominio, la racionalidad, la eficiencia y la resistencia o aguante; mientras que la feminidad abarca aspectos como la sumisión, la dependencia, la afectividad y las características ligadas al rol reproductivo y del cuidado de otros (Heilbrun, 1981; Díaz Loving et al., 2007).

Empero, con base en hallazgos sistemáticos, la postura bipolar y unidimensional cambió eventualmente a la idea de que son atributos de personalidad autónomos (Díaz Loving, Rocha \& Rivera, 2007), por lo que pueden coexistir en cada individuo independientemente de si se es hombre o mujer. Esto se debe a que la cultura sufre transformaciones y por ende impacta y modifica la forma en la cual se integran y conforman los diferentes estereotipos, incluyendo los que se vinculan con la instrumentalidad (conjunto de características ligadas a la masculinidad que refieren la competiti- vidad, independencia y orientación al logro), así como las que se vinculan con la expresividad (características relacionadas con la feminidad y que involucran la expresión de afectos, el cuidado de otro y la dependencia afectiva). La transformación de la cultura implica la posibilidad de que las personas manifiesten una mayor diversidad en la combinación de estas características. Sumado a esto, cada etapa de la vida conlleva una serie de modificaciones sobre el significado social que tiene el individuo en función de su género (Fernández, 1996; Galambos, Almeida \& Petersen, 1990; Ussher, 1991). Así, las personas pueden ser femeninas, masculinas o bien indiferenciadas o andróginas (Bem, 1981). Esto último implica que las personas pueden presentar diferentes grados de estereotipamiento y rigidez alrededor de su género, comportándose de manera sumamente tradicional o alejarse de dichos parámetros.

En el análisis de los diversos indicadores involucrados en la salud de las personas, se observa que las mujeres tienen mayor expectativa de vida que los varones, situación que parece depender del síntoma que se analice y de la fase de vida en la cual se encuentra la persona (Walters, McDonough \& Strohschein, 2002). La explicación de las diferencias que se detectan entre la salud de los hombres y las mujeres se ha abordado desde diversos niveles (Walsh et al., 1995 en Kawachi, Kennedy, Gupta \& Prothrow-Stith, 1999); 
aquellos relacionados con las diferencias genéticas (modelo biomédico), lo que tiene que ver con las diferencias en un nivel intrapsíquico e interpersonal, enfatizando las diferencias en la personalidad, afrontamiento, autoeficacia, etcétera (modelo psicosocial), la visión que contempla los factores de riesgo (epidemiológico) y el aspecto que analiza el impacto de los procesos culturales, económicos y políticos.

Algunos autores (Matud, Rodríguez, Marrero \& Carballeira, 2002) enfatizan el impacto que factores sociopsicológicos como la masculinidad y la feminidad tienen en la salud de las personas. De esta manera, las investigaciones señalan que los valores asociados a los estereotipos de género tradicionales y que se vinculan a las características de personalidad femeninas como la sumisión y la dependencia pueden favorecer la aparición de trastornos mentales como la depresión (Weissman \& Klerman, 1977). Seguidamente, la masculinidad y la androginia positiva en diversos estudios han sido asociados a una mejor salud mental (Bassof \& Glass, 1982). De manera consistente se valora que la racionalidad, el pensamiento lógico, la expresión de ira y la conducta asertiva pueden favorecer la resolución y el enfrentamiento de situaciones estresantes. Aunque cabe señalar que Good, Sherron \& Dillon (2000) enfatizan los costos de estas características en la salud de los hombres. Entre los costos se han señalado el peso que tiene sobre el hombre el estar condicionado estrictamente a demostrar dominancia y hegemonía, poder y autoridad, sin espacio para la debilidad y con un estricto control emocional, lo cual impide relaciones interpersonales constructivas, y termina teniendo un efecto negativo entre los que se incluyen la soledad, la conducta antisocial y la agresividad (Matud, Rodríguez, Marrero \& Carba1leira, 2002).

El patrón de estereotipos se vincula a que de manera consistente las mujeres tienden a deprimirse con mayor frecuencia y a enfrentar mayor ansiedad, en tanto los hombres suelen vincularse más a trastornos de personalidad antisocial y de abuso de sustancias (Kessler et al., 1994). Estas diferencias parecen vincularse con el tipo de personalidades, ya que, por ejemplo, la descarga emocional -más típica de las mujeresse asocia a la depresión y la de los hombres a trastornos de enojo vinculados a enfermedades cardiacas (Billings \& Moos, 1981). En tanto, algunas características -asociadas típicamente a los hombres- tales como la objetividad, el centrarse en el problema y buscar soluciones, hace que la aparición de trastornos mentales sea menos frecuente o al menos reduce la experiencia depresiva.

En general, parece que la instrumentalidad y la expresividad son rasgos valiosos que al ser socialmente deseables y combinarse en una misma persona favorecen que sean más felices y que tengan un mejor ajuste psicológico 
y emocional (Hegelson \& Fritz, 1999). Sin embargo, aún no es claro cómo estas características se vinculan directamente a los trastornos de salud mental. Por lo que desde una perspectiva de género, el abordaje de los problemas de salud es un campo de investigación relativamente reciente y todavía poco explorado (Guibert, Prendes, González \& Valdés, 1999). Sin embargo, conforme aparecen trabajos en el enfoque de género, se afianza la idea acerca del significado que la sociedad confiere al hecho de ser hombre y mujer, y cómo esto influye diferencialmente en la salud de las poblaciones al originar comportamientos y actitudes que determinan diferentes grados de riesgo. De hecho, la literatura científica ha señalado diferenciales empíricos entre hombres y mujeres en relación con necesidades especiales de atención, riesgos específicos de atención ligados a actividades difundidas como masculinas o femeninas, percepciones de enfermedad, conductas de búsqueda de atención, y grado de acceso y de control ejercidos por las personas sobre los recursos básicos para la protección de la salud. De acuerdo a la OMS (2002), un amplio número de estudios proveen evidencia de que diferencias de género contribuyen significativamente en la prevalencia de depresión y desórdenes de ansiedad, falta de autoestima y otros trastornos sitúan a las mujeres como la población vulnerable (Ryba \& Hopko, 2012). Estas investigaciones muestran que las di- ferencias socialmente construidas entre hombres y mujeres en cuanto a sus roles y responsabilidades, estatus y poder, interactúan con las diferencias biológicas y contribuyen a diferencias en la naturaleza de la salud mental ante los problemas de la vida cotidiana.

Cabe enfatizar la importancia de un modelo holístico con componentes bioevolutivos, socioculturales e individuales en la concepción del fenómeno de salud. Según Afifi (2007), rara vez el componente biológico (sexo) determina por sí solo las consecuencias en la salud. Determinantes sociales (que incluyen el género) interactúan para exacerbar puntos biológicos vulnerables. De hecho, Astbury (2006, en Afifi, 2007) encontró que las diferencias por género en los desórdenes mentales van más allá de diferencias en los índices de frecuencia de ocurrencia de uno $u$ otro trastorno. De acuerdo con algunos autores (Hawton, Rodham, Evans, \& Weatherall, 2002; Parker \& Roy, 2001), durante la adolescencia, las mujeres tienen una mayor prevalencia de depresión y desórdenes alimenticios, y también se involucran más en conductas suicidas en comparación con los varones. Durante la etapa adulta, las mujeres presentan mayor cantidad de trastornos afectivos, mientras que los hombres se involucran en mayor cantidad de abuso de sustancias (Linzer, Spitzer, Kroenke et al., 1996).

Al introducir la variable sociocultural en el estudio del desarrollo de géne- 
ro, es necesario identificar la educación formal, el empleo y otros movimientos hacia el desarrollo personal de las mujeres como detonantes de cambios sociales e individuales. Dado que el sexo y el género interactúan con otros determinantes sociales, el esfuerzo adicional de las mujeres como consecuencia de la cantidad de roles que realizan tiene como consecuencia una sensibilidad distinta de cada uno de los géneros ante eventos estresantes en la vida. Ahora, las mujeres tienen mayor probabilidad de entrar en estados depresivos o ansiosos dado que aunque enfatizan la realización de papeles que implican niños, casa y reproducción, también tienen que jugar cada vez más roles que involucran tanto actividades financieras, como trabajo y otras actividades (Nazroo, 2001). Como resultado, las mujeres en general están más expuestas a eventos negativos de vida que los hombres (Dalgard et al., 2006). En particular, las mujeres que carecen de apoyo social, que están expuestas a ciertos eventos estresantes de vida son más vulnerables que los hombres que no tienen apoyo social. Además, Dalgard et al. (2006) indican que los niveles de depresión de las mujeres no se explican por diferencias de cantidad de apoyo social o vulnerabilidad, sino por los atributos predominantemente emocionales que tienen para liderar con las situaciones. Con la misma preocupación, el rol de los factores de personalidad y las diferencias de género han sido estudiados por Goodwin y
Gotlib (2004), quienes encontraron que los niveles de neuroticismo, el cual es significativamente más alto en las mujeres, puede moderar la asociación entre el sexo femenino y un elevado riesgo de depresión en etapas adultas de la vida. Otros estudios en esta misma línea encontraron que las mujeres son más propensas a ser diagnosticadas con algún tipo de ansiedad que los hombres (Simonds \& Whiffen, 2003). De esta manera, la proporción de mujeres que experimentan ansiedad por sí sola o ansiedad en combinación con depresión tiende a ser mayor que en los hombres (Silverstein, 2002).

Ahora bien, cuando los cambios en los roles van aunados a cambios en las competencias que las personas tienen para enfrentar los conflictos de la vida, la expectativa de salud se modifica. En México, cuando se pretende analizar y entender los patrones diferenciales de malestar o bienestar, se alude a múltiples factores involucrados, entre los que se encuentran el hecho de pertenecer a uno u otro sexo y los atributos de instrumentalidad y expresividad que presenta cada individuo. Se ha sugerido que en la medida en que una persona realiza diferentes roles y desarrolla características de personalidad adecuadas para enfrentarlos con éxito, esta obtiene más beneficios, particularmente en torno a su bienestar emocional y psicológico (Barnett, 1998). En el caso de las mujeres, se ha aludido al hecho de que conforme enfrentan cambios y se involucran en 
más roles, se asocia a una mayor salud emocional en comparación con mujeres que se mantienen en un solo rol (sobre todo si se trata del estereotipo femenino) (Moen, Robinson, \& Dempster, 1995).

Ahora bien, es fundamental considerar que cualquier periodo de transición presenta falta de certeza e incluye ciertos niveles de ansiedad. Rocha (2008) reporta hallazgos en los que por una parte la permanencia en rasgos, roles y actitudes tradicionales conlleva, tanto a hombres como a mujeres, a la experiencia de cierto malestar que se ve reflejado en el tipo de estados anímicos reportados. Empero, al mismo tiempo, ante la adopción de roles y rasgos no convencionales ni estereotipados también se hace presente este malestar. Es posible que aunque hombres y mujeres experimenten mayor bienestar general cuando incorporan actividades y rasgos no tradicionales, algunos de estos al estar enfrentando el statu quo conducen a un estado anímico de malestar.

El estudio de Rocha (2008) destaca que aparentemente sigue existiendo una presión social fuerte en torno a que hombres y mujeres realicen actividades altamente estereotipadas. Las actividades y rasgos asociados "tradicionalmente" a cada género conllevan un significado positivo o negativo que facilita el hecho de que ante ciertos roles o características ambos sexos experimenten malestar, tal como otros autores lo han señalado (Moen et al., 1995). En este sentido, aun si un rol es parte de un estereotipo, dados los cambios sociales e ideológicos actuales, es factible que ambos géneros contrasten los beneficios y costos de un rol de esta naturaleza, por lo que en este sentido las connotaciones afectivas de los estereotipos resultan relevantes como consideración ante la posibilidad de que las personas "híbridas" en cuestión de rol de género, no experimenten malestar, es decir los andróginos. Con la finalidad de valorar el efecto que tiene la adopción de distintos patrones de instrumentalidad y expresividad positiva y negativa sobre la salud mental de hombres y mujeres, se realizó la presente investigación.

\section{Método}

\section{Participantes}

Participaron 200 personas voluntarias, 100 hombres y 100 mujeres. El rango de edad fue de 18 a 57 años, con una media de 22.45 (D.S.=8.98). El 96\% de los participantes tenía la licenciatura y $94 \%$ eran solteros.

\section{Instrumentos}

\section{Masculinidad-feminidad}

Se usó del inventario realizado por Díaz Loving, Rocha Sánchez y Rivera Aragón (2007). La versión corta de dicha escala consta de 65 adjetivos que refieren las dimensiones de instrumen- 
talidad y expresividad. La escala tiene un formato de respuesta tipo Likert de cinco opciones que van desde "mucho", hasta "nada", en términos de posesión de cada adjetivo o característica. Se pidió a los participantes que respondan de la manera en la cual ellos se perciben y no como les gustaría ser.

Los factores que conforman dicha escala son:

\section{Instrumentalidad}

a) Cooperativo $(\alpha=.90)$.- En este factor se observa la relación de características instrumentales positivas socialmente deseables para los hombres y para las mujeres, haciendo ver una personalidad precavida, trabajadora, formal, activa, ordenada, cumplida, responsable y organizada.

b) Orientado al logro $(\alpha=.85)$.- En este otro factor se presentan características ligadas a versiones individuales, donde se enfatiza la competencia individual encaminada al progreso personal: autosuficiente, persistente, competitivo, íntegro, constante, ambicioso, competente, etcétera.

c) Egocéntrica $(\alpha=.67)$.- El tercer factor es una visión positiva de la instrumentalidad, la cual hace alusión a características tales como ser atrevido, arriesgado, arrojado, valiente.

d) Machismo $(\alpha=.85)$.- Factor que hace alusión a características como ser aprovechado, tosco, agresivo, avorazado, violento, patán. e) Autoritaria-manipuladora $(\alpha=.77)$.Las características que conforman este factor son: ser orgulloso, manipulador, vengativo, terco.

f) Rebelde social $(\alpha=63)$.- Se compone de características donde se encuentra la descortesía, el desinterés, la irreflexividad, lo cual mostraría a una persona que no manifiesta interés en las relaciones interpersonales.

\section{Expresividad}

a) Afectivo-afiliativo $(\alpha=.85)$.- Personalidad afiliativo-afectiva, donde predominan los rasgos como ser amoroso, cariñoso, dulce, tierno, fiel, cálido. Estos rasgos son valorados y socialmente deseables, para un favorecimiento de su interacción social, encaminadas al cuidado y bienestar común.

b) Romántico-soñador $(\alpha=.79)$.- El siguiente factor hace referencia a una expresividad romántico soñadora; los rasgos son ser soñador, romántico, emocional, sensible. Apunta más a un patrón conductual.

c) Emotivo-negativo-egocéntrica $(\alpha=.83)$. - En el lado oscuro de la expresividad se presenta emotivonegativo-egocéntrica, que se reviste como inestable, mentiroso, quejumbroso, burlón, metiche.

d) Vulnerable-emocional $(\alpha=.76)$.- $\mathrm{El}$ siguiente factor responde a una expresividad vulnerable-emocional, donde se encuentran características 
como ser celoso, preocupado, infantil, miedoso, llorón.

e) Control-pasivo-negativa $(\alpha=.72)$.- Él último factor que se describe en el instrumento es el de control-externopasivo-negativa; donde encontramos abnegación, dependencia, sumisión, conformidad, entre otras. Características, estas últimas, asociadas a los estereotipos femeninos.

2. Inventario de salud mental (Estrada, Rocha, Rivera, Díaz Loving, manuscrito no publicado)

Está formado por noventa reactivos que exploran la salud mental del individuo, utilizando una escala de respuesta tipo Likert de cinco niveles (desde totalmente en desacuerdo hasta totalmente de acuerdo). La escala consta de doce dimensiones:

a) Disforia.- Implica sensaciones de malestar e irritabilidad con un estado de ánimo depresivo-ansioso, aumentando la reactividad y las manifestaciones físicas de tensión $(\alpha=.87)$.

b) Asertivo-seguro de sí mismo.- Comprende la sensación de seguridad y autoconfianza, así como una autoestima positiva, manifiesta la capacidad de resolver conflictos y mantener el control $(\alpha=$.79).

c) Narcisismo.- Congrega la idea de superioridad y egocentrismo, así como una opinión exagerada de sí mismo $(\alpha=.75)$. d) Inseguro-dependiente.- Refiere la necesidad afectiva de ser importante y reconocido por los otros, hipersensibilidad a la crítica y rasgos de baja autoestima e inseguridad. Refleja desconfianza y la creencia de que los demás albergan intenciones maliciosas, con una falta de interacción espontánea $(\alpha=.80)$.

e) Paranoide.- Indica características vinculadas a la sensación de desconfianza y agresión por parte de otros, junto con la idea delirante de persecución y engaño $(\alpha=$.72).

f) Comportamientoantisocial.-Conduc tas excluyentes en la interacción social, manifestando una incapacidad para interactuar adecuadamente con el medio $(\alpha=.67)$.

g) Pensamiento fantasioso.- Planeación $\mathrm{y}$ anhelo de logro de metas, que a veces pueden ser poco aceptadas debido a su irrealidad $(\alpha=.58)$.

h) Obnubilación.- Comprende el enturbiamiento de la conciencia caracterizado por una disminución del estado de alerta, los procesos mentales y la asociación entre ellos, pudiéndose cometer errores de juicio y omisiones, así como tener pensamientos confusos e inconexos $(\alpha=.65)$.

i) Depresión.- Refleja la condición emocional caracterizada por un estado de ánimo abatido, con aflicción, desesperanza y rasgos de ansiedad y angustia $(\alpha=.52)$.

j) Comportamiento apático.- Condición emocional caracterizada por un bajo 
interés y motivación en cualquier actividad o proyecto $(\alpha=.51)$.

k) Soledad.- Condición emocional de aislamiento y exclusión social $(\alpha=52)$.

1) Trastornos de la memoria.- Altera ciones vinculadas al déficit de la capacidad de atención y recuerdo que produce distracción, olvido y baja concentración $(\alpha=.62)$.

\section{Resultados}

En primera instancia se realizó un análisis de correlación de Pearson con la finalidad de establecer el patrón de salud mental de mujeres con diferentes perfiles de instrumentalidad y expresividad. En el caso de las mujeres se observó que la instrumentalidad y expresividad socialmente deseables se asocian con diversos factores de salud mental. De manera específica, existe una correlación negativa entre el factor instrumental cooperativo (ser cumplida, responsable, ordenada) y factores que indican la falta de salud mental, como disforia, narcisismo, inseguridad, obnubilación, depresión, apatía, soledad, trastornos de la memoria y ser antisocial; y una correlación positiva con el factor asertivo-seguro. En términos del factor instrumental orientado al logro (ser competente, tenaz, cabal, inteligente) poseer dichas características disminuye la probabilidad de cualquier indicio de problemas de salud mental, y por el contrario, aumenta el nivel de asertivo seguro, con el cual se correla- ciona positivamente, es decir, aquellas mujeres competentes, tenaces, son asertivas-seguras. Incluso el factor de instrumental egocéntrico (ser arriesgada, atrevida, valiente), el cual sigue siendo positivo pero con un sobreinterés en la resolución de las necesidades personales, se correlaciona negativamente con los factores inseguro, paranoide, antisocial, obnubilación y depresión en la escala de salud mental, mostrando así su potencial de promoción de la salud mental.

En términos de las características de personalidad instrumentales, socialmente indeseables, se encuentra que aquellas mujeres con características instrumentales autoritarias (mandonas, orgullosas, dominantes) solo mostraron correlaciones positivas con el factor disforia. Lo cual hace pensar que utilizar estilos de interacción autoritarios protege hasta cierto punto a las mujeres de un entorno cada vez más hostil en su ingreso al mundo del trabajo abierto. En el caso del factor instrumental rebelde-social (ser descortés, desagradecida, desatenta e irreflexiva), este sí se correlaciona positivamente con varios factores que indican una falta de salud mental. Así aparecen calificaciones altas en disforia, narciso, inseguro, paranoide, antisocial, obnubilación, depresión, apático y trastornos de la memoria; y tiene una correlación negativa con el factor asertivo-seguro de la escala de salud mental, por lo tanto, 
aquellas mujeres descorteses, desagradecidas y desatentas no son asertivasseguras.

En cuanto a las características expresivas positivas en mujeres y su relación con la salud mental, resulta interesante que no existan correlaciones significativas entre el factor expresivo afiliativo, es decir, ser amorosa, cariñosa y tierna, con los factores de la escala de salud mental. En el caso del factor romántico-soñador (sensible, soñadora, emocional) únicamente encontramos correlaciones positivas con los factores disforia, narciso, inseguro, fantasioso y obnubilación, es decir, las mujeres sensibles, soñadoras y emocionales se muestran disfóricas, narcisas, inseguras, fantasiosas y obnubiladas.

Para el factor vulnerable emocional en mujeres (ser llorona, chillona, miedosa), se encontraron correlaciones positivas con los siguientes factores de la escala de salud mental: disforia, narciso, inseguro, paranoide, antisocial, fantasioso, obnubilación y trastornos de la memoria; en contraparte, se encontró una correlación negativa con el factor asertivo-seguro, lo cual indica que las mujeres lloronas, chillonas, miedosas, tenderán a ser disfóricas, narcisistas, inseguras, paranoides, antisociales, fantasiosas, obnubiladas y mostrarán trastornos en la memoria. Para el factor control externo pasivo-negativo en mujeres (ser conformista, indecisa, sumisa, penosa) se encontraron correlaciones positi- vas con todos los factores de la escala de salud mental, excepto con el factor asertivo-seguro con el cual tiene una correlación negativa; es decir, las mujeres conformistas, sumisas y penosas no se mostrarán asertivas ni seguras.

En el caso de los hombres es evidente que las características masculinas y femeninas positivas tienen un efecto protector y promotor de salud mental. De manera contraria, las características negativas dirigen a la persona a una mayor predisposición hacia cierto tipo de trastornos. En primer lugar, se observa que la presencia de una instrumentalidad positiva orientada al logro se vincula con el hecho de ser más asertivos y claramente menos narcisistas, menos paranoides, menos fantasiosos, menos obnibulados, menos solitarios y menos apáticos. Por el contrario, el trayecto hacia la insalubridad mental se ve zanjado en aquellos que son descorteses, poco atentos, débiles, chillones, quejumbrosos, y que se muestran dejados, sumisos y conformistas. De hecho, se puede indicar que el patrón conductual de las personas que muestran rasgos de masculinidad y feminidad negativa es en sí una marca de falta de estabilidad y desarrollo mental. Finalmente, cabe señalar que estos patrones de instrumentalidad y feminidad positiva y negativa afectan directamente la salud y el bienestar de las personas, pero a la vez señalan el tipo de relaciones interpersonales, afectando directamente su satisfacción con sus parejas y con sus 


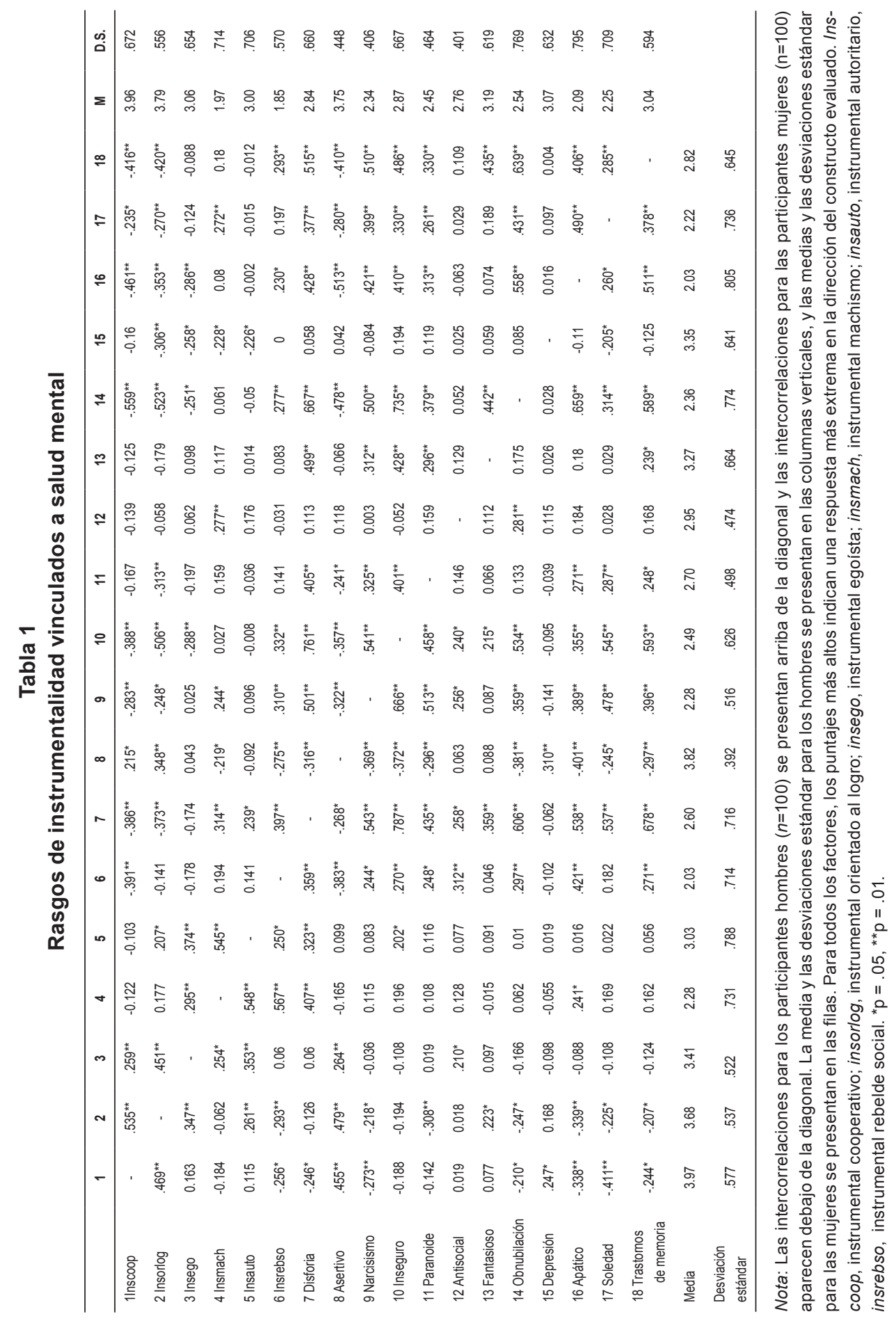




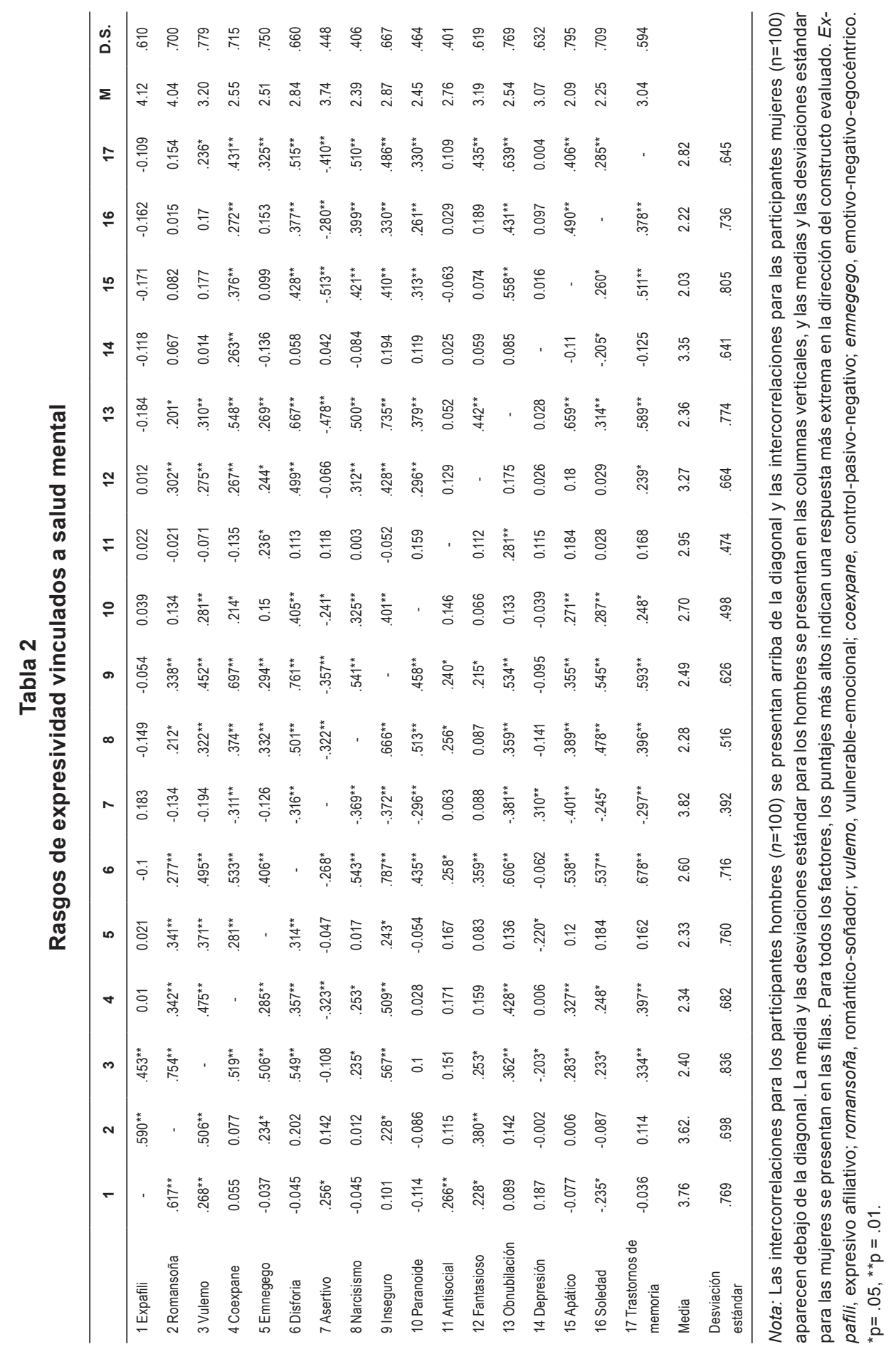


Rolando Díaz Loving, Sofía Rivera Aragón \& Pedro Wolfgang Velasco Matus

Tabla 3

Análisis de regresión 'enter' que predice la salud mental desde los rasgos de masculinidad-feminidad para hombres

\begin{tabular}{|c|c|c|c|c|c|c|c|}
\hline Variables predictoras & $\mathbf{R} 2$ & $\Delta \mathrm{R} 2$ & & $\mathbf{F}$ & $\Delta \mathrm{F}$ & \multirow{2}{*}{\multicolumn{2}{|c|}{ IC $95 \%$}} \\
\hline MODELO & .515 & .515 & & $8.479^{* * *}$ & $8.479 * * *$ & & \\
\hline & & B & $\beta$ & $\mathbf{t}$ & & LI & LS \\
\hline Instrumental cooperativo & & -.986 & -.182 & -1.778 & & -2.087 & .116 \\
\hline $\begin{array}{l}\text { Instrumental orientado } \\
\text { al logro }\end{array}$ & & -1.996 & -.301 & $-2.939^{* * *}$ & & -3.346 & -.646 \\
\hline Instrumental egoísta & & .215 & .038 & .407 & & -.835 & 1.264 \\
\hline Instrumental autoritario & & -.631 & -.122 & -1.243 & & -1.639 & .378 \\
\hline Expresivo afiliativo & & -1.565 & -.262 & $-2.388^{* *}$ & & -2.868 & -.263 \\
\hline Romántico-soñador & & 1.097 & .209 & 1.583 & & -.280 & 2.473 \\
\hline Vulnerable-emocional & & .722 & .153 & 1.229 & & -.446 & 1.889 \\
\hline Control-pasivo-negativo & & 1.083 & .212 & 1.857 & & -.076 & 2.241 \\
\hline Instrumental machismo & & .828 & .162 & 1.615 & & -.191 & 1.847 \\
\hline $\begin{array}{l}\text { Instrumental rebelde } \\
\text { social }\end{array}$ & & -.129 & -.020 & -.215 & & -1.322 & 1.064 \\
\hline $\begin{array}{l}\text { Emotivo-negativo- } \\
\text { egocéntrico }\end{array}$ & & .019 & .004 & & & -.963 & 1.002 \\
\hline
\end{tabular}

Nota: $\mathrm{N}=200 . \mathrm{IC}=$ Intervalo de confianza $\quad{ }^{* *} \mathrm{p}<.01,{ }^{* * *} \mathrm{p}<.001$

relaciones (véanse las tablas 1 y 2).

Finalmente, un análisis factorial de segundo orden sobre las dimensiones de salud mental revela un constructo general donde menor asertividad y seguridad y mayor cantidad de todas las dimensiones de patología mental conforman un solo factor. Al computar este conglomerado de todas las partes se procedió a ver el impacto de la masculinidad y la feminidad sobre los niveles de insalubridad mental. Para ello se realizó un análisis de regresión tipo 'enter', con la finalidad de conocer el impacto que tienen los rasgos de masculinidad- feminidad sobre la salud mental en hombres y mujeres. En la tabla 3 se muestra el análisis de regresión para hombres en el cual se incluyeron los factores en el siguiente orden: instrumental cooperativo, instrumental orientado al logro, instrumental egoísta, instrumental autoritario, expresivo afiliativo, romántico-soñador,vulnerable-emocional,controlpasivo-negativo, instrumental machismo, instrumental rebelde social y emotivo-negativo-egocéntrico. Se observa que el modelo explica el $51,5 \%$ de la varianza $F(11,88)=8.47, p=.000$.

En la tabla 4 se muestra el análisis de 
Tabla 4

Análisis de regresión 'enter' que predice la salud mental desde los rasgos de masculinidad-feminidad para mujeres

\begin{tabular}{|c|c|c|c|c|c|c|c|}
\hline Variables predictoras & R2 & $\Delta \mathrm{R} 2$ & & $\mathbf{F}$ & $\Delta \mathrm{F}$ & \multirow{2}{*}{\multicolumn{2}{|c|}{ IC $95 \%$}} \\
\hline MODELO & .387 & .387 & & $5.042^{* * *}$ & $5.042^{* * *}$ & & \\
\hline & & B & $\beta$ & $\mathbf{t}$ & & LI & LS \\
\hline Instrumental cooperativo & & -1.444 & -.212 & $-2.011^{*}$ & & -2.872 & -.017 \\
\hline $\begin{array}{l}\text { Instrumental orientado } \\
\text { al logro }\end{array}$ & & -.296 & -.040 & -.361 & & -1.923 & 1.331 \\
\hline Instrumental egoísta & & .438 & .058 & .584 & & -1.052 & 1.927 \\
\hline Instrumental autoritario & & .784 & .156 & 1.314 & & -.402 & 1.971 \\
\hline Expresivo afiliativo & & .685 & .134 & 1.064 & & -.594 & 1.963 \\
\hline Romántico-soñador & & -.343 & -.061 & -.494 & & -1.723 & 1.037 \\
\hline Vulnerable-emocional & & 1.789 & .376 & $3.016^{* * *}$ & & .610 & 2.968 \\
\hline Control-pasivo-negativo & & 1.312 & .226 & $2.056^{*}$ & & .044 & 2.579 \\
\hline Instrumental machismo & & .091 & .017 & .135 & & -1.239 & 1.420 \\
\hline $\begin{array}{l}\text { Instrumental rebelde } \\
\text { social }\end{array}$ & & 1.207 & .219 & $1.989^{*}$ & & .001 & 2.413 \\
\hline $\begin{array}{l}\text { Emotivo-negativo- } \\
\text { egocéntrico }\end{array}$ & & 1.328 & .255 & $2.138^{*}$ & & 2.563 & .094 \\
\hline
\end{tabular}

Nota: $\mathrm{N}=200 . \mathrm{IC}=$ Intervalo de confianza $\quad{ }^{*} \mathrm{p}<.05,{ }^{* *} \mathrm{p}<.01,{ }^{* * *} \mathrm{p}<.001$

regresión para mujeres en el cual se incluyeron los factores en el siguiente orden: instrumental cooperativo, instrumental orientado al logro, instrumental egoísta, instrumental autoritario, expresivo afiliativo, romántico-soñador, vulnerable-emocional, control-pasivonegativo, instrumental machismo, instrumental rebelde social y emotivonegativo-egocéntrico. Se observa que el modelo, explica el $38,7 \%$ de la varianza $\mathrm{F}(11,88)=5.04, \mathrm{p}=.000$.

\section{Discusión}

Las transformaciones económica, educativa y política han incidido en la creación de normas sociales que han impulsado el ingreso de la mujer al campo de la autoridad, lo cual se refleja en la posesión de ciertas características instrumentales acompañadas de aspectos tales como mostrarse responsable, independiente y hasta arriesgada en el campo positivo y abusiva en el campo negativo, al mismo tiempo que ya no siempre conservan las características expresivas positivas y por tanto no es necesario el mostrarse atenta, cortés o reflexiva. El movimiento, sin duda, tie- 
ne efectos sobre el desarrollo personal y económico de las mujeres. Un ámbito adicional que se toca en la presente investigación es el efecto de la movilidad sobre las relaciones interpersonales armoniosas y la salud mental de las mujeres. Como se puede observar en las correlaciones, la mujer posmoderna que choca con el papel tradicional e incorpora los aspectos negativos de la instrumentalidad, como ser egoísta, autoritaria y rebelde, hace que claramente sea percibida como antisociable, más apática, tenga rasgos narcisistas, paranoides, problemas para pensar con claridad y de memoria y carezca de competencias asertivas. En concordancia con otras investigaciones, la instrumentalidad negativa en las mujeres tiene un efecto devastador sobre su propia salud mental (Matud, Rodríguez, Marrero \& Carballeira, 2002).

Empero, como se ha presentado, la instrumentalidad tiene un lado constructivo y socialmente deseable que incluye la cooperación y la orientación al logro, tener estos atributos en general para las mujeres parece ofrecer la posibilidad de un protector de salud, ya que en la medida en que son instrumentales positivas, existe una disminución de muchos de los trastornos y esto parece coincidir con la idea de la masculinidad como predictora de salud mental. Lo interesante es la relación que existe entre los rasgos instrumentales y expresivos socialmente deseables. Cuando las mujeres se mueven hacia la parte construc- tiva de la instrumentalidad y no pierden la parte expresiva positiva, esto las protege de incorporar la parte negativa de la masculinidad. En este sentido, es posible pensar que las mujeres, aunque incursionan en el ámbito de la instrumentalidad, no abandonan del todo la expresividad y esto favorece la presencia de una personalidad más andrógina y por tanto promotora de salud mental (Bem, 1993).

Sin embargo, a pesar de lo anterior, si las mujeres no desarrollan la parte positiva de la masculinidad, llama la atención que la sensibilidad coincida con características de salud mental como la disforia, el narcisismo, la inseguridad, las fantasías y la obnubilación. Parecería, entonces, que lo femenino, cuando no es controlado por la racionalidad de la instrumentalidad conlleva -por su sensibilidad emocional- mayor riesgo de trastornos e inestabilidad. Esta situación coincide con la manera en la cual las investigaciones han planteado hasta ahora la instrumentalidad positiva como más cercana a la salud mental, mientras que la expresividad en todas sus acepciones se aleja de esta (Weissman \& Klerman, 1977). Inclusive, algunos autores (Billings \& Moos, 1981) han reportado que el manejo de la descarga emocional en las mujeres es responsable de la gravedad con la que se viven los trastornos y la sintomatología de estos. En este sentido, parecería coincidir el hecho de que los hombres que son románticos y expresivos manifiesten como 
trastorno el pensamiento fantasioso, es decir, que solamente bajo la "irracionalidad" pueden encubrirse las emociones y la expresión de afectos. Esta situación hace suponer que unas características en el interior de lo positivo son mejores que otras; finalmente, la emotividad no tiene por qué ser vista como algo negativo cuando es regulada, lo cual obedece a la visión sociocultural de los hombres y las mujeres sobre sus propios roles y características (Rocha, 2004).

Lo realmente interesante es que por encima de los efectos principales de la masculinidad y la feminidad están las interacciones, ya que la combinación de aspectos positivos de ambas dimensiones puede garantizar una mayor salud mental, en parte porque esto implica más flexibilidad hacia la manera como se comportan las personas y a la vez una mejor adaptación al medio y las circunstancias (Bem, 1993; Green \& Kenrick, 1994). De hecho, se ve en los resultados que la presencia de instrumentalidad positiva y de expresividad positiva funcionan como protectores $y$ promotores de un mejor estado de salud mental. En contraste, el rebelde social, la feminidad negativa (vulnerabilidad emocional) y el control externo pasivo negativo, características que implican tanto aspectos instrumentales como expresivos en su versión oscura, contribuyen en la aparición de más trastornos de la salud mental. Ciertamente, lo que se puede concluir es que gran parte de la presencia de trastornos se debe a la manera como se enfrentan las situaciones y los estresores, y en este proceso los hombres han sido socializados para ser menos sensibles y más racionales y concretos, centrándose en la tarea, en tanto las mujeres han sido socializadas para la parte afectiva (Rocha, 2004), favoreciendo que la rumiación y la exacerbación emocional favorezcan una mayor incidencia de ciertos trastornos (Nolen-Hoeksema, 1991; Nolen-Hoeksema, Larson \& Grayson, 1999). Empero la realidad más allá de la brecha genérica, lo que parece evidente es que cuando se manifiesta un patrón conductual de personas que muestran rasgos de masculinidad y feminidad negativa, estas se convierten en sí en una marca de falta de estabilidad y desarrollo mental. En este sentido, Bem (1993) sugiere que lograr la androginia implica tiempo y flexibilización asociada a la madurez intelectual y a la toma de conciencia de la brecha ideológica entre los géneros.

Finalmente, al observar las regresiones, es claro que para las mujeres es particularmente dañino desarrollar atributos de masculinidad negativa, como la rebeldía social, y conservar el lado negativo de la expresividad, como la vulnerabilidad emocional, el control pasivo negativo y el egocentrismo emotivo negativo. Su salud mental se derivará fundamentalmente de desarrollar los rasgos de instrumentalidad cooperativa, que implica ser responsable, independiente, trabajadora. Por su parte, 
en los hombres es fundamental seguir siendo orientados al logro, no incorporar la parte negativa de la instrumentalidad, cuyos efectos negativos en la salud no aparecen en esta investigación pero sí han sido reportados con anterioridad (Good, Sherron \& Dillon, 2000), sobre todo para los tiempos de hoy, si no desarrollan características expresivas constructivas, que les permitan tener relaciones interpersonales armoniosas, su salud mental será más precaria.

Como corolario, cabe señalar que estos patrones de instrumentalidad y feminidad positiva y negativa inciden directamente en la salud y el bienestar de las personas, y en el tipo de relaciones interpersonales, afectando directamente su satisfacción con sus vidas y con sus relaciones (Green \& Kenrick, 1994, Hegelson \& Fritz, 1999). Como consecuencia, parece necesario promover la androginia positiva (responsables y cariñosos) para garantizar mayor salud mental.

\section{RefERENCIAS}

Afifi, M. (2007). Gender differences in mental health. Singapore Medical Journal, 48 (5), 385-391.

Barnett, R. C. (1998). Toward a review and reconceptualization of the work/ family literature. Genetic, Social, and General Psychology Monographs, 124(2), 125-182.

Bassof , E. S., \& Glass, G. V. (1982). The relationship between sex roles and mental health: a meta-analysis of twenty-six studies. The Counseling Psychologis, 10, 105-112.

Bem, S. L. (1981). Gender Schema Theory: A Cognitive account of sextyping. Psychological Review, 88, 354-364.

Bem, S. L. (1993). The lenses of Gender: transforming the debate on sexual inequality. New Haven, CT: Yale University.

Billings, A. G., \& Moos, R. H. (1981). The role of coping responses and social resources in attenuating the impact of stressful life events. Journal of Behavioral Medicine, 4, 139-157.

Dalgard, O., Dowrick, C., Lehtinen, V., et al. (2006). Negative life events, social support and gender differences in depression: A multinational community survey with data from the ODIN study. Social Psychiatry and Psychiatric Epidemiology, 41, 444-451.

Díaz Loving, R., \& Rocha, T. (2008). La masculinidad y la feminidad a través del ciclo vital: un estudio comparativo. En S. Rivera Aragón, R. Díaz Loving, R. Sánchez Aragón $\&$ I. Reyes Lagunes (Eds.), La psicología social en México, XII (pp. 213220). México: Asociación Mexicana de Psicología Social.

Díaz Loving, R., Rocha, T., \& Rivera, S. (2004). Elaboración, validación y estandarización de un inventario para evaluar las dimensiones atribu- 
tivas de instrumentalidad y expresividad. Revista Interamericana de Psicología, 38(2), 263-276.

Díaz Loving, R., Rocha, T., \& Rivera, S. (2007). La instrumentalidad y expresividad desde una perspectiva psico-socio-cultural. México: Miguel Ángel Porrúa.

Estrada J., Rocha T., Rivera S., \& Díaz Loving R. Inventario de salud mental. Manuscrito inédito.

Erickson, E. (1968). Identity, youth and crisis. Nueva York: Norton.

Fernández, J. (1996). Varones y mujeres: Desarrollo de la doble realidad del sexo y del género. Madrid: Psicología Pirámide.

Galambos, N. L., Almeida, D. M., \& Petersen, A. C. (1990). Masculinity, femeninity and sex roles attitudes in early adolescence: Exploring gender intensification. Child Development, 61, 1905-1914.

Good, G. E., Sherron, N. B., \& Dillon, M. G. (2000). Masculine gender role stressors and men's health. En R. M. Eisler y M. Hersen (Eds.), Handbook of gender, culture and health. Mahwah, Estados Unidos: LEA.

Goodwin, R., \& Gotlib, I. (2004). Gender differences in depression: The role of personality factors. Psychiatry Res, 126, 135-142.

Green, B. L., \& Kenrick, D. T. (1994). The attractivenes of gender-typed traits at different relationships levels: Androgynous characteristics may be desirable after all. Personality and Social Psychology Bulletin, 20, 244-253.

Guibert, W., Prendes, M., González, R., \& Valdés, E. (1999). Influencia en la salud del rol de género. Revista $\mathrm{Cu}$ bana de Medicina General Integral, 15(1), 7-13.

Hawton, K., Rodham, K., Evans, E., \& Weatherall, R. (2002). Deliberate self-harm in adolescents: self-report survey in schools in England. BMJ, 23, 1207-1211.

Hegelson, V. S., \& Fritz, H. L. (1999). Unmitigated agency and unmitigated communion: distinctions from agency and communion. Journal of Research in Personality, 33, 131-158.

Heilbrun, A. B. (1981). Human sex-role behavior. Nueva York: Pergamon Press.

Jayme, M., \& Sau, V. (2004). Psicología diferencial del sexo y el género. Barcelona: Icaria.

Kawachi, I., Kennedy, B. P., Gupta, V., \& Prothrow-Stith, D. (1999). Women's status and the health of women and men: A view from the states. Social Science and Medicine, 48, 21-32.

Kessler, R. C., McGonagle, K. A., Zhao, S., Nelson, C. B., Hughes, M., Eshleman, S., Wittchen, H., \& Kendler, K. S. (1994). Lifetime and 12 month prevalence of DSM-III-R psychiatric di- 
Rolando Díaz Loving, Sofía Rivera Aragón \& Pedro Wolfgang Velasco Matus

sorders in the United States. Archives

of General Psychiatry, 51, 8-19.

Linzer, M. Spitzer, R., Kroenke K., et al. (1996). Gender, quality of life and mental disorders in primary care: Results from the PRIME-MD. American Journal of Medicine, 101, 526-533.

Matud, A. M. P., Rodríguez, W. C., Marrero, Q. R., \& Carballeira, A. M. (2002). Psicología del género: Implicaciones en la vida cotidiana. Madrid: Biblioteca Nueva.

Medina, M. (2007). Masculinidades en la era de la razón. Propuesta para el estudio de la identidad y autorrepresentaciones en Suecia y Sudamérica a principios del 1800. En M.C. Medina, E. Domínguez, \& R. Icaza-Garza (Eds.), Género y globalización en América Latina, (pp. 139150). Göteborg: IberoameriKansKa Institutet, Göteborgs Universitet.

Moen, P., Robinson, J., \& Dempster, M. D. (1995). Caregiving and women's well-being: A life course approach. Journal of Health and Social Behavior, 36(3), 259-273.

Nazroo, J. Y. (2001). Exploring gender differences in depression. Psychiatric Times, 18(3).

Nolen-Hoeksema, S. (1991). Responses to depression and their effects on the duration of depressive episodes. Journal of Abnormal Psychology, 100, 569-582.
Nolen-Hoeksema, S., Morrow, J., \& Grayson, C. (1999). Explaining the gender differences in depressive symptoms. Journal of Personality and Social Psychology, 77, 1061-1072.

OMS/WHO (2002). Gender and mental health. Departamento de Género y Salud de la Mujer. Recuperado el 19 de mayo del 2012, de http://whqlibdoc.who.int/gender/2002/a85573.pdf

Parker, G., \& Roy, K. (2001). Adolescent depression: A review. Australian Journal of Psychiatry, 35, 572-580.

Ryba M. M., \& Hopko D. R. (2012). Gender differences in depression: Assessing mediational effects of overt behaviors and environmental reward through daily diary monitoring. Depression Research and Treatment. 1-9. doi:10.1155/2012/865679.

Rocha T. (2008). Identidad de género y estado anímico en hombres y mujeres. En S. Rivera Aragón, R. Díaz Loving, R. Sánchez Aragón \& I. Reyes Lagunes (Eds.), La psicología social en México, XII (pp. 663-670), México: Asociación Mexicana de Psicología Social.

Rocha, S. T. (2004). Socialización y cultura: El impacto de la diferenciación entre los sexos. (Tesis de doctorado inédita). Universidad Nacional Autónoma de México, Facultad de Psicología.

Silverstein, B. (2002). Gender differences in the prevalence of somatic ver- 
sus pure depression: A replication. American Journal of Psychiatry, 159, 1051-1052.

Simonds, V., \& Whiffen, V. (2003). Are gender differences in depression explained by differences in co-morbid anxiety? Journal of Affect Disorders, 77, 197-202.

Spece, J., \& Helmreich, R. (1978). Masculinity and feminity: Their psychological dimensions, correlates and antecedents. Austin: University of Texas Press.

Spence, J. (1993). Gender-related traits and gender ideology: Evidence for a multifactorial theory. Journal of Personality and Social Psychology, 64(4), 624-635.

Stoller, J. R. (1968). Sex and gender: The development of masculinity and feminity. Karnac: Maresfield Library.

Ussher, J. (1991). La psicología del cuerpo femenino. Madrid: Arias Montano.

Walters, V., McDonough, P., \& Strohschein, L. (2002). The influence of work, household structures, and social, personal and material resources on gender differences in health: A analysis of the 1994 Canadian national population health survey. Social Science and Medicine, 54, 677-692.

Weissman, M. M., \& Klerman, G. K. (1977). Sex differences and the epidemiology of Depression. Archives of General Psychiatry, 34, 98-111. 